\title{
Novel air leak test using surfactant for lung surgery
}

\author{
Hee Chul Yang', Hye Young Chang ${ }^{2}$
}

${ }^{1}$ Center for Lung Cancer, Research Institute and Hospital, National Cancer Center, Goyang, Korea; ${ }^{2}$ Bio-Medical Research Institute, Kyungpook National University, Daegu, Korea

Contributions: (I) Conception and design: HC Yang; (II) Administrative support: HC Yang; (III) Provision of study materials: All authors; (IV) Collection and assembly of data: HC Yang; (V) Data analysis and interpretation: All authors; (VI) Manuscript writing: All authors; (VII) Final approval of manuscript: All authors.

Correspondence to: Hee Chul Yang, MD, PhD. Center for Lung Cancer, Research Institute and Hospital, National Cancer Center, 410-769 Ilsan-ro 323, Ilsan, Goyang, Korea. Email: yang@ncc.re.kr.

Background: Prolonged air leak is the most common complication after pulmonary resection surgery. Water submersion test (WST) has been used to check for air leak. However, it is cumbersome under the circumstances of video-assisted thoracic surgery (VATS). This study aimed to devise a new air leak detection method that is suitable for the VATS.

Methods: We decided to utilize the properties of the surfactants to overcome the disadvantages of WST. To find the optimal surfactant, ex-vivo porcine lung experiments were prepared with a custom-made large glass vessel mimicking a human thoracic cavity. A fresh lung was put inside the glass vessel and connected with the ventilator. We made a needle injury on the lung surface and dropped various kinds of liquid surfactants to create air bubbles during the lung ventilation. The appearances of bubbles were recorded through $5 \mathrm{~mm}$ thoracoscope.

Results: Considering the bubble forming ability, Pluronic F-127 solution (PF127), a well-known non-toxic and non-ionic colorless surfactant, was chosen as candidate substance. To find the optimal condition, various concentrations of PF127 (30\%, 25\%, 20\%, 15\%, 10\%) were tested. Greater than 20\% concentration of PF127 were not feasible due to its high viscosity; the bubbles kept increasing in size without popping and blocked the thoracoscopic vision. The 10\% PF127 did not form any bubbles. On the contrary, the 15\% PF127 formed bubbles that are 1-2 cm in size with dynamic movement allowing for clear visibility of the air leak point. We finally made a green colored $15 \%$ PF127 by mixing an indocyanine green to increase its visibility. All of the components in the solution are FDA approved and permissible to be used in the human body.

Conclusions: Our bubble solution can easily detect the air leak even in small quantities and is expected to be useful in VATS with limited vision. However, in order for its full-scale clinical use, its safety in the human body must be verified.

Keywords: Air leak; video-assisted thoracic surgery (VATS); uniportal; Pluronic F-127; indocyanine green; bubble

Submitted Jul 30, 2018. Accepted for publication Sep 26, 2018.

doi: $10.21037 /$ jtd.2018.11.34

View this article at: http://dx.doi.org/10.21037/jtd.2018.11.34

\section{Introduction}

Prolonged air leak (PAL) that lasts for more than 5 days is the most common complication after pulmonary resection surgery. About $8-10 \%$ of the patients undergoing pulmonary lobectomy are related to this (1). PAL may lead to a longer hospital stay, require pleurodesis with irritating substances or reoperation, heighten patient's pain, increase medical costs, and potentially cause serious complications such as empyema (2). One of the best preventive methods is to confirm and block the air leak points before closing the wound in the operating room. For decades, a water submersion test (WST) has been used to check for air leak as a final operative step. Over the years, however, we have 


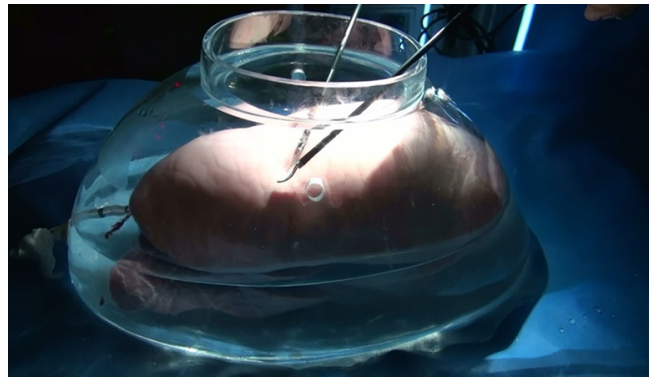

Figure 1 Ex-vivo porcine lung experiment mimicking water submersion test of video-assisted thoracic surgery.

come to the era of video-assisted thoracic surgery (VATS), which inevitably made it more difficult to perform the WST. Moreover, several researchers insisted that VATS did not decrease the frequency of the PAL compare to open thoracotomy (3). The exact air leak point detection via the thoracoscope is challenging because the vision of the scope is blurred or hindered by the water in the thoracic cavity and the inflated lung itself during the WST. Also, surgical drapes are likely to get wet because the water overflows out of the patient's thoracic cavity during the lung ventilation. Therefore, we developed a new method to replace the WST being suitable for VATS.

\section{Methods}

The preconditions to devise the new air leak detection method are as follows: (I) obstruction of the vision should be minimized; (II) air leak point should be clearly confirmed; (III) the utilization must be easy under VATS; (IV) the substance should be harmless for human; (V) the substance should be economical.

Considering the preconditions, we decided to utilize the properties of surfactants. For an ex-vivo experiment with porcine lungs, we made a large glass vessel mimicking a human thoracic cavity (Figure 1). A fresh porcine lung (right or left lung) was put inside the glass vessel and connected with the ventilator via an endotracheal tube. The lung was mechanically ventilated under the peak inspiratory pressure $20 \mathrm{cmH}_{2} \mathrm{O}$ with positive end-expiratory pressure $5 \mathrm{cmH}_{2} \mathrm{O}$. We made an 18-gauge needle injury on the lung surface and dropped various kinds of liquid surfactants to create air bubbles during the lung ventilation. The appearances of bubbles during the inflation period were recorded through 5-mm thoracoscope. Unlike the WST method, each lobe was examined separately by just instilling surfactant solutions over the lung surface not to pour it.

\section{Results}

Ex-vivo porcine lung experiments were performed more than 50 times. Initially, it was to find out which surfactant was suitable for air leak detection from the lung and tested many of the surfactants on the market. Among the many substances tested, Pluronic ${ }^{\circledR}$ F-127 (PF127, poloxamer 407, Sigma P2443) was closest to the ideal bubble conditions, which is a well-known non-ionic surfactant comparably non-toxic to human. We made a solution by mixing the PF127 powder and Phosphate-buffered saline (PBS, $\mathrm{pH}$ 7.4). To completely dissolve PF127 powder which has properties of high molecular weight (average $12.6 \mathrm{kDa}$ ) and thermosensitivity into PBS, we cooled the solution to a temperature of $4{ }^{\circ} \mathrm{C}$ using the refrigerator and stirred it.

Various concentrations of PF127 solutions (10\%, 15\%, $20 \%, 25 \%, 30 \%)$ were tested to determine the optimal concentration for air leak detection. Through an ex-vivo experiment, we found that solutions with higher than $20 \%$ concentration of PF127 were not feasible due to its high viscosity. The high viscosity bubbles kept increasing in size without popping and blocked the vision of the thoracoscope. The solution with $10 \%$ concentration of PF127 did not form any bubbles. On the contrary, the $15 \%$ solution generated consistent small bubble streams with 1 or $2 \mathrm{~cm}$ in size. The dynamic movement of small bubbles made it possible to detect the exact location of the air leak. However, it was difficult to indicate where the solution was sprayed. Therefore, we mixed $500 \mathrm{~mL}$ of $15 \%$ PF127 solution with $25 \mathrm{mg}$ of indocyanine green to make the solution stand out. We named it "Yang's bubble solution" (Figure 2).

\section{Discussion}

We set out to develop a novel air leak test that is appropriate for the VATS. As a result of testing several surfactants, the green colored $15 \%$ PF 127 solution was found to be the most suitable for detecting air leak from the lung. In our simulation, approximately $60 \mathrm{~mL}$ of the Yang's bubble solution was sufficient to inspect one whole porcine lung under the guidance of thoracoscopy. In other words, the $60 \mathrm{~mL}$ was the total amount of the bubble solution to test the one-sided lung partially and sequentially by instilling it. We believe that Yang's bubble solution can make air leak detection easier and more accurate. However, the 


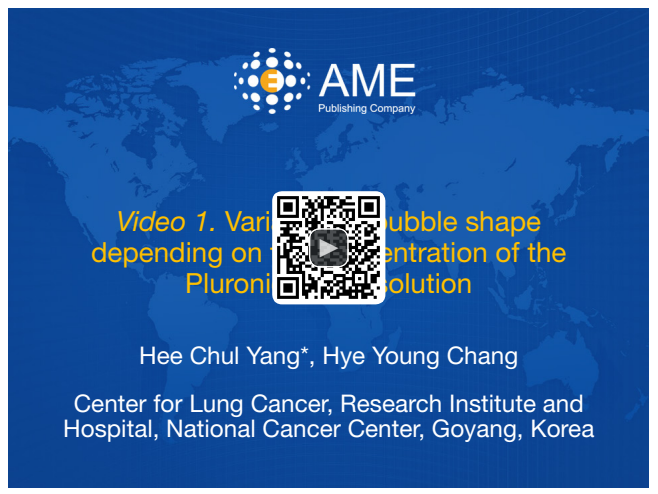

Figure 2 Variation of bubble shape depending on the concentration of the Pluronic F-127 solution (4).

Available online: http://www.asvide.com/article/view/29194

randomized prospective study should be conducted as to whether this will ultimately reduce the incidence of PAL.

PF127, main ingredient, is obviously more expensive than normal saline or distilled water. However, it is not too difficult to obtain and is affordable enough to make it commercialize. Moreover, it should be diluted, and less than $100 \mathrm{~mL}$ is needed to examine the entire one lung. These conditions will help reduce the manufacturing cost. However, in the present situation, it can only offer the possibility of economic feasibility. Since it is still in the animal testing phase, there is no question that a clinical trial will be needed in the future to discuss the cost effectiveness mentioned above.

PF127 has a distinctive feature of thermo-reversible hydrogels (5). It means that PF127 will form gels above $10^{\circ} \mathrm{C}$ when used at concentrations of $18-50 \%$. It will re-liquefy when cooled to below $10{ }^{\circ} \mathrm{C}$. Although $15 \%$ PF127 remained unchanged as liquid during the ex-vivo air leak test, it should be verified through an in-vivo experiment to confirm how this material changes in the warm thoracic cavity.

All the constituents of Yang's bubble solution were approved by FDA and have been used in medical practice, drug manufacturing process or cell culture $(6,7)$. However, even if each component is safe, preclinical animal toxicity experiment is needed to guarantee the safety of the mixture. And then, its safety in the human body must be verified for its clinical use.

\section{Acknowledgements}

Funding: This work was supported in part by Seoul
National University Bundang Hospital research fund (No. 11-2011-027).

\section{Footnote}

Conflicts of Interest: The authors have no conflicts of interest to declare.

Ethical Statement: The study was approved by the Institutional Animal Care and Use Committee (IACUC number: BA1112-095/077-01).

\section{References}

1. Brunelli A, Pompili C, Dinesh P, et al. Financial validation of the European Society of Thoracic Surgeons risk score predicting prolonged air leak after video-assisted thoracic surgery lobectomy. J Thorac Cardiovasc Surg 2018;156:1224-30.

2. Wood DE, Lauer LM, Layton A, et al. Prolonged length of stay associated with air leak following pulmonary resection has a negative impact on hospital margin. Clinicoecon Outcomes Res 2016;8:187-95.

3. Paul S, Altorki NK, Sheng S, et al. Thoracoscopic lobectomy is associated with lower morbidity than open lobectomy: a propensity-matched analysis from the STS database. J Thorac Cardiovasc Surg 2010;139:366-78.

4. Yang HC, Chang HY. Variation of bubble shape depending on the concentration of the Pluronic F-127 solution. Asvide 2018;5:932. Available online: http://www.asvide. com/article/view/29194

5. Nie S, Hsiao WL, Pan W, et al. Thermoreversible Pluronic F127-based hydrogel containing liposomes for the controlled delivery of paclitaxel: in vitro drug release, cell cytotoxicity, and uptake studies. Int J Nanomedicine 2011;6:151-66.

6. Danson S, Ferry D, Alakhov V, et al. Phase I dose escalation and pharmacokinetic study of pluronic polymerbound doxorubicin (SP1049C) in patients with advanced cancer. Br J Cancer 2004;90:2085-91.

7. Escobar-Chávez JJ, López-Cervantes M, Naïk A, et al. Applications of thermo-reversible pluronic F-127 gels in pharmaceutical formulations. J Pharm Pharm Sci 2006;9:339-58.

Cite this article as: Yang HC, Chang HY. Novel air leak test using surfactant for lung surgery. J Thorac Dis 2018;10(12):6472-6474. doi: 10.21037/jtd.2018.11.34 